\title{
Vers une politique de développement intégré
}

Oléagineux, Corps Gras, Lipides. Volume 8, Numéro 3, 179, Mai - Juin 2001, Editorial

Auteur(s) : Jean-Claude ICART, .

Résumé : Les analyses présentes dans cette livraison relèvent de quelques problématiques contribuant à des titres divers à la réflexion aujourd'hui nécessaire sur le développement.

\section{ARTICLE}

Les analyses présentes dans cette livraison relèvent de quelques problématiques contribuant à des titres divers à la réflexion aujourd'hui nécessaire sur le développement ${ }^{1}$.

La question de la qualité sanitaire des productions est un de ces nouveaux sujets touchant aujourd'hui de très près les pays exportateurs en développement et tout particulièrement les pays du continent africain. Compliquant leur accès aux marchés internationaux, le renforcement des réglementations adoptées au niveau de I'OMC ou de I'Union européenne en matière de sécurité sanitaire (le taux des mycotoxines admis par l'Union européenne a été ainsi amené aux limites de détection analytique !) pourrait entraîner leur mise à l'écart définitive du commerce mondial, si des mesures adaptées ne sont pas rapidement mises en œuvre.

La démarche qualité filière, initiée par le Cirad dans le cas de l'arachide de bouche au Sénégal et impliquant une maîtrise des caractéristiques de la production (matériel végétal, pratiques agricoles) et de la transformation (auto-contrôle (HACCP), traçabilité), ouvre ici un nouveau champ d'action à la recherche pour le développement.

Concernant en l'occurrence un secteur où $85 \%$ de la production sont assurés par le système paysannal "traditionnel ", cette démarche se révèle également profondément tributaire de la volonté politique des pouvoirs publics, seuls à même de décider des bases législatives et réglementaires et de la mise en place des pratiques d'inspection, d'analyses et de veille scientifique nécessaires. Soit une situation où " l'allégement de l'Etat ", longtemps prescrit par les institutions internationales, pourrait se révéler, s'il a été mené à son terme, non moins préjudiciable que son pesant interventionnisme antérieur ${ }^{2}$.

Notons au passage combien Bruxelles, semble ici convaincu de la nécessité de mettre en œuvre une politique d'aide à la mise aux norme de qualité (voir l'article de P. Vialatte).

Ce rôle des politiques publiques en tant que facteur " d'évolution des filières " est d'ailleurs le second thème dominant de ce dossier. Les analyses de l'économie du soja en Chine et au Brésil révèlent, sous le couvert d'une volonté de mondialisation affichée, les contours bien présents et les effets non moins sensibles des nouvelles politiques agricoles de ces deux acteurs importants. 
Symétriques dans leurs objectifs - panoplies de mesures discrètes en faveur du commerce extérieur pour le Brésil, importation de grains et transformation sur place pour la Chine, entraînant une crise de la trituration du soja au niveau mondial -, ces politiques (quoiqu'encore largement indéchiffrable dans ses fondements dans le cas de la Chine $^{3}$ ) intéressent à plus d'un titre les producteurs, opérateurs et autres acteurs du développement.

Elles devraient en particulier, constituer une bonne incitation à revisiter l'idée de " politique agricole communautaire ${ }^{4}$ et à comprendre la nécessité de rouvrir, de par les aléas futurs du marché mondial qu'elles laissent entrevoir, le dossier « protéines » (voir l'article de Y. Dronne).

\section{Notes:}

${ }^{1}$ Sujet également au centre du récent rapport du CNJA, « Paysan du monde : le prix de notre avenir ».

${ }^{2}$ Voir le rapport « Développement » du CAE, Paris : Documentation française, 2000.

${ }^{3}$ Voir sa décision récente et surprenante de limiter les importations de soja transgénique. La Tribune, 14 juin 2001.

${ }^{4}$ Tombée en désuétude depuis 1992 selon le CNJA. 